\title{
Ontology Based Method for Sentiment Examination
}

\author{
Puninder Kaur, Ruchi Mittal
}

\begin{abstract}
In recent years, sentiment analysis became an important concept which is used to extract an important feature or text mining according to our interest. It is very difficult to judge the polarity or the review of particular product. This problem is solved by ontology. Ontology is a formal and shared specification of a particular domain. It is a large semantic network used to extract a domain specific concept or domain specific features of a particular product. Ontology contains concept which means domain and sets of objects or we can say instance of concept and relationship between various objects. Ontology based approach is classification of opinion and feature-based approach. Ontology gives domain specific features along with the hieratical relationship between them. Ontology generates semantic graphs and tree for showing relationship between different domains and entities. The relationship can be done on the basis of nodes and edges connections. We use some contextual lexicons which are used to provide the opinion about particular domain like SentiWordNet, SenticNet, WordNet etc. This survey aims to provide an insight on Opinion target, Opinion lexical and Aspect based Polarity detection, sentiment aggregation.
\end{abstract}

Keywords: ontology, aspect level polarity, semantic network, SentiWordNet, SenticNet, sentiment analysis.

\section{INTRODUCTION}

The ontology is branch of metaphysics which deals with "what exists" and "how it exists". It is concerned with the braches like entity, concept, relationship and properties. In the mid of 1970 Artificial intelligence (AI) was introduced. With using of AI the computational model ontology was created. In 1980 we use ontology to both theory and component using knowledge based system. In 1990 the ontology it is connect with widely cited web pages. In 1993 the classes and inheritance features are developed. Each ontology is concerns with the entity, attributes, classes and relations between different objects. It is a kind of dictionary or methods why which we can analyze our opinion. The first English ontology is OED (oxford English Dictionary) which is introduced in 1988. It is first automatic dictionary which is related with ontology. Ontology is divided in various categories according to their working and performance like domain ontology, hierarchal and upper ontology. Ontology uses two languages RDF and OWL. With the using of ontology we can creates large graphs and trees.

Revised Manuscript Received on November 08, 2019.

Puninder Kaur, Chitkara University Institute of Engineering and Technology, Chitkara University, Punjab, India

Ruchi Mittal, Chitkara University Institute of Engineering and Technology, Chitkara University, Punjab, India

* Corresponding Author: ruchi.mittal@chitkara.edu.in

\section{BACKGROUND}

\section{Sentiment Analysis}

Sentiment analysis (SA) is used to extract an important feature about a particular product. There is a very large amount of data that is available on the internet and it is difficult to access all the data at the same time. What sentiment analysis does is to extract the relevant data about a particular product thereby providing an opinion about the product, additionally, it also tells us about its polarity i.e. whether the text is positive, negative or neutral [1]. SA is also called opinion mining, survey mining, evaluation extraction or state of mind investigation. It is the assignment of recognizing, separating and grouping suppositions, feelings and dispositions concerning diverse points, as communicated in textual information [2]. SA helps in accomplishing different objectives and has a very diverse set of applications , for instance, observing public opinion with respect to a political development, advertising insight, estimation of consumer loyalty, movie reviews, sales and purchase predictions and so on [3][4]. Sentiment analysis has a very critical application in the field of social media especially when existing customers post their opinion regarding their experiences with a product or service. Such information (sentiments) can be used by marketers to formulate more effective marketing strategies [5].

The sentiment analysis is divided into three levels [7][8] namely, (1) document level; (2) sentence level; and (3) feature (aspect) level.

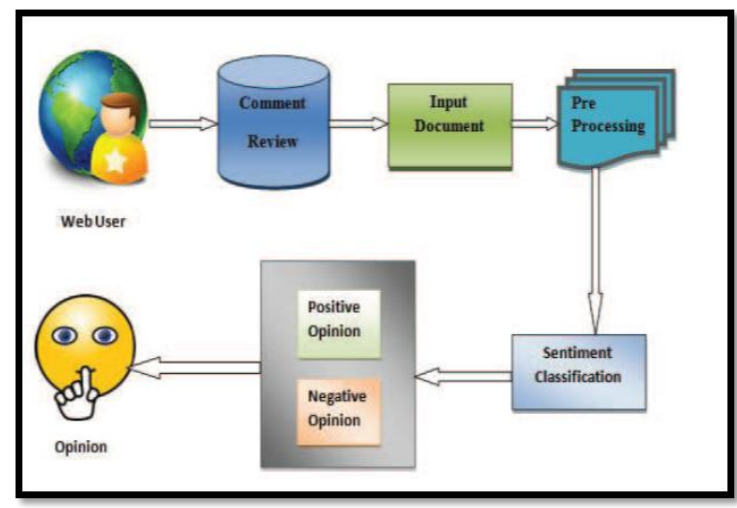

Figure1: Sentiment analysis concept [7]

\section{Ontology}

Ontology is an explicit, formal and shared specification of a domain which has specific logical properties. According to Studer et al. (1998) an ontology can be defined as an "explicit, machine-readable specification of a shared conceptualization". The specification is explicit because 
it summarizes the properties and relationship among the particular concepts [10]. Ontology consists of product, product features, sentiment words extract the product feature on the basis of sentiment lexicon and it associates its polarity [11][12]. Ontology use two types of languages: traditional language and web based ontology (RDF, OWL) [13].Ontology is a large semantic network which has large amount of commonsense concept is used for various inferences from text. The ontology output in the context of sentiment analysis can be shown by the semantic graphs or semantic tree.[17][18][19].We have some types of ontology which gives us the properties of a particular concept[20][55][56] namely (1) upper ontology, (2) domain ontology, (3) interface ontology, and (4) process ontology. Upper ontology is a top level ontology which consists of general terms such as property, attributes and relationship between them. Domain ontology is like a concept which is relevant to the particular area like in the case of mobile phone the camera quality of phone is very good so the domain is camera in this case. Interface ontology is a medium or way by which various concepts and attributes are related to each other. Process ontology consist inputs, outputs, conditions, sequence, information and opinion of concept.

The ontology is divided in to three categories according to their relations [34]:

1. Hierarchical relation: hierarchical relationship is that relation which is in the particular hierarchy means tells us about parent child relation like Located-Near, Has-A, Made-Of, Is-a, Inherit-From etc[35][36].

2. Synonymous relation: synonym relation is that relation which has some similar types of words or some related words and we detect these kind words for example synonym, Conceptually-Related-To[37][38]

3. Functional relation: functional words inform us about property and purpose of a concept and these relations are Used-For, Capable-Of,

Defined-As, Has-Property[39][40][41].

These all relations are used as an edge which is used to connect two or more different nodes or concepts. While creating a relation between different concepts the relation can be one-to-one and one-to-many.

ConceptNet ontology has some primary relation which is used to connect different concepts [42] and these relations are:

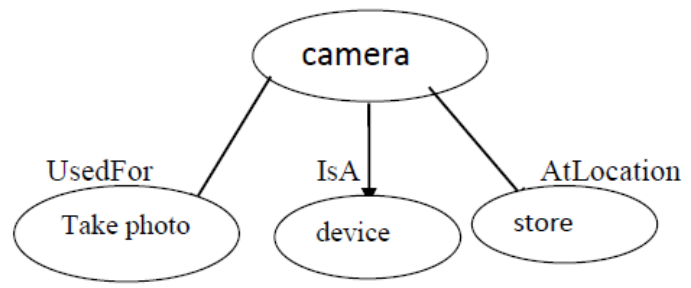

Figure 2. Sample Ontology of Camera

This figures shows following types of relation:

- Camera is UsedFor take photos.

- Camera IsA device.

- Camera is AtLocation store.

According to Mukherejee and Joshi, 2013, ontology is due to the following reasons:

1. Ontology predicts the relationship between various concepts for building ontology. For example, relations like partof, hasa, madeof, usedfor. Like camera is UsedFor take photos.[43]

2 . The relations creates by ontology does not requires labeling for review a particular product. [21][37][44][45].

3 . Ontology is closed class of well-defined relations so it can be applied or suitable for all domains [21][46][47].

There is some task that is to be performed by using sentiment analysis using ontology.

1. Opinion target which means we have to indentify entity i.e. what we want. Suppose I want to purchase a mobile phone of Samsung then in this case my target is about mobile phone [48][49].

2. Opinion lexicon is online dictionary which is basically gives us contextual information. SentiWordNet is online available dictionary which is taken from WordNet[38][50][51].

3. Aspect level polarity which is find outcome that means polarity tells us about the product is positive, negative and neutral and determine the polarity of a particular product.[40[41][53]

For example: "The camera quality of phone is good". So here according to this example opinion target i.e. domain is mobile. Opinion lexical is used to find out common word i.e. "good" and aspect level polarity checks polarity which means it gives positive response[54][55].

\section{LITERATURE REVIEW}

Padmaja et al. (2013) tell us about the sentiment analysis (SA) and opinion mining and how to identify the features of a particular product and extract their polarity. It uses the NLP (natural language processing) tools kits for common sense knowledge. Nithish et al. (2013) used the OWL (web ontology language) format in SA to create domain ontology, extract relevant reviews, and outline the process of SA. Jagtap et al. (2013) conducted SA using machine learning approach and data classification models which they suggest gives better results. Kontopoulos et al. (2013) and Vivekanandan et al. (2014) followed frequency based, relational based and model based approach is used. Accuracy can be measured on the basis of recall, precision and F-measure. Ravi et al. (2015) it provide additional insights into sentiment analysis where sentiment lexicons are generated and summarize the opinion of a particular product., Kolkur et al. (2015) in their paper generate different levels and conduct an analysis on all three levels. Devika, Sunitha \& Ganesh et al. (2016) conducted a sentiment analysis are on twitter data and other social websites. Machine learning and lexical based approach are used and compared which is very similar to the study conducted by Mäntylä et al. (2018)

Kreutzer et al (2016) discuss about wordNet and sentiwordNet which is largest lexicon database and gives the contextual lexical. Borth et al. (2013) in their study look at visual sentiment ontology and use of SentiBank as a novel visual concept detector for detecting an image. Mukherjee et al. (2013) describes the process followed in their study as follows: construct ontology, check sentiment lexicon using sentiwordnet, select attributes and calculate the feature specific concept, make a tree based on the ontology after this sentiment aggregate approach by combining all polarity and last is detect the negative opinion. Najmi et al. (2013) use conceptNet. In this paper the 
work is divided into two parts: in first part generate a common sense knowledge using conceptNet ontology and after that the relation of product can be done by conceptOnto ontology. The conceptOnto ontology convert conceptnet into RDA format. Here wordNet is replaced by babelNet. Tsai et al. (2014) use two types of ontology, firstly ontology mining and secondly product ontology. Using the ontology aspect based sentiment analysis can be detected. Social analytical methodology is used for sentiment analysis. Fuzzy ontology also is used. Cambria et al. (2013) in their study the knowledge based approach is used for sentimental analysis. It provides the brief information about and task performed by sentiment analysis. In Saif et al. (2014), lexicon adaption approach is used for sentiment analysis. F-measure is used for check the accuracy for different data sets. Cross et al. (2014) use fuzzy ontology in their study and for better performance OWL language is used to extend the language with new construction methods. In this paper ontology based approach is used for text classification and compared this technique with previous one Wójcik et al. (2014). Ahire et al. (2014) conducted a study on sentiment lexicons where three sentiment lexicons are used i.e. DO-CAL, SentiWordNet and Sentiment TreeBank.
Yang et al. (2015) used FOBPRM which refers to feature ontology based review miner which is basically used for review. Dou et al. (2015), Agarwal, et al. (2015) Agarwal et al. (2016) used SA in the later years. Agarwal et al. (2016) used Naïve Bayes, Support Vector machine and machine learning algorithms for features and for polarity detection. Common sense knowledge is used in the form of ConceptNet based ontology. Thakor \& Sasi et al. (2016) used GATE software which gave results in tags form. Balachandran et al. (2016). . In 2016, several high quality research papers were published in the area i.e Ullah, Said, Saleem, Jaafar et al. (2016), Hussein et al. (2016), Arunachalam et al. (2016), Tamilselvam et al. (2016) Zhou et al. (2016), Cambria et al. (2016), Alhojely, S. et al. (2016) and Salas-Zárate et al. (2016) amongst others. Substantial research has been carried forward to 2017 and 2018, for instance Icarte et al. (2017), Tamilselvam et al. (2017), Ali et al. (2017), Ma et al. (2018), Maurya et al. (2018), Dragoni et al. (2018), Cambria et al. (2018), Mirtalaie et. al. (2018), Vyas et. al.(2018) Schouten et. al.(2018), Pouriyeh et. al.(2018).

SUMMARY OF SENTIMENTAL BASED ONTOLOGY

\begin{tabular}{|c|c|c|c|}
\hline ontology & Approach & Advantages & Future scope \\
\hline $\begin{array}{l}\text { Visual sentiment } \\
\text { ontology }\end{array}$ & $\begin{array}{l}\text { SentiBank Visual } \\
\text { detector }\end{array}$ & $\begin{array}{l}\text { Detect visual images and gives } \\
\text { the sentiment prediction using } \\
\text { the SentiBank visual } \\
\text { detection... }\end{array}$ & $\begin{array}{l}\text { In feature cross domain } \\
\text { performance detector can be used } \\
\text { for depth study of concept. Extend } \\
\text { VSO and Sentibank for video } \\
\text { applications also. }\end{array}$ \\
\hline $\begin{array}{l}\text { ConceptNet based } \\
\text { Ontology }\end{array}$ & $\begin{array}{l}\text { Weakly supervised } \\
\text { approach. }\end{array}$ & $\begin{array}{l}\text { Ontology tree is used for } \\
\text { sentiment analysis. }\end{array}$ & $\begin{array}{l}\text { More number of domains can be } \\
\text { developed. }\end{array}$ \\
\hline Fuzzy ontology & $\begin{array}{l}\text { Fuzzy logic and Fuzzy } \\
\text { reasoning }\end{array}$ & $\begin{array}{l}\text { Web ontology language(OWL) } \\
\text { and automatic tools used }\end{array}$ & $\begin{array}{l}\text { Some ontologies are incorporate } \\
\text { to some standard tools in future } \\
\text { more ontologies are used. }\end{array}$ \\
\hline ConceptOnto & $\begin{array}{l}\text { It is based on ConceptNet } \\
\text { and RDF Format... }\end{array}$ & $\begin{array}{l}\text { It use Conceptnet which extract } \\
\text { domain name and its properties } \\
\text { and convert it into RDF. }\end{array}$ & $\begin{array}{l}\text { Using RDF it convert triple based } \\
\text { data set in future more number of } \\
\text { data set can be added. }\end{array}$ \\
\hline Semantic web ontologies & $\begin{array}{l}\text { Ontology based } \\
\text { association Rule mining } \\
\text { and Web ontology } \\
\text { Language is used. }\end{array}$ & $\begin{array}{l}\text { Semantic Web ontology helps } \\
\text { us to guiding mining process } \\
\text { and reduce search space. }\end{array}$ & Performance can be improved. \\
\hline Web ontology & $\begin{array}{l}\text { Machine learning } \\
\text { approach }\end{array}$ & $\begin{array}{l}\text { It helps us for polarity } \\
\text { detections. }\end{array}$ & $\begin{array}{l}\text { Other methods can be used for } \\
\text { improve performances }\end{array}$ \\
\hline ConcepNet ontology & $\begin{array}{l}\text { Opinion Lexicons are } \\
\text { used. }\end{array}$ & $\begin{array}{l}\text { WordNet are expands and } \\
\text { polarity is also evaluates. }\end{array}$ & $\begin{array}{l}\text { Semantic lexicons can be enriched } \\
\text { for opinion mining. }\end{array}$ \\
\hline $\begin{array}{l}\text { Financial domain } \\
\text { Ontology }\end{array}$ & $\begin{array}{l}\text { SentiwordNet is used for } \\
\text { feature polarity detection }\end{array}$ & $\begin{array}{l}\text { It is used to detect the financial } \\
\text { news. }\end{array}$ & $\begin{array}{l}\text { In future we can develop } \\
\text { supervised learning method for } \\
\text { automatic creation of ontology. }\end{array}$ \\
\hline Dynamic ontology & $\begin{array}{l}\text { NLP is used for text } \\
\text { classification. }\end{array}$ & $\begin{array}{l}\text { Dynamic ontology } \\
\text { classification is fast for text } \\
\text { classification and gives us } \\
\text { opinion about particular } \\
\text { domain. }\end{array}$ & $\begin{array}{l}\text { We need more research for remove } \\
\text { ambiguities for text classification }\end{array}$ \\
\hline Feature based ontology & $\begin{array}{l}\text { Review based algorithm } \\
\text { is used. }\end{array}$ & $\begin{array}{l}\text { Its gives the opinion of a } \\
\text { product and also used for } \\
\text { sentiment aggregation. }\end{array}$ & $\begin{array}{l}\text { Building ontology is difficult task } \\
\text { so for future we can develop } \\
\text { automatic method for detecting } \\
\text { polarity and opinion mining. }\end{array}$ \\
\hline $\begin{array}{l}\text { Ontology summarization } \\
\text { method }\end{array}$ & $\begin{array}{l}\mathrm{RDF} / \mathrm{S} \text { is used for } \\
\text { ontology summarization. }\end{array}$ & $\begin{array}{l}\text { It is used for ontology } \\
\text { summarization technique using } \\
\text { graph based methods. }\end{array}$ & $\begin{array}{l}\text { Ontology summarization should } \\
\text { be flexible so in future other } \\
\text { methods can use. }\end{array}$ \\
\hline
\end{tabular}




\section{CONCLUSION}

This survey is conducted because it is very difficult to know the opinion about a particular product. So, at this time text mining is required when text is classified then polarity cannot be assimilated easily so solve this problem we use ontology. Ontology is kind of dictionary which provides the polarity words. We have various types of ontology and algorithms. In this survey we are analyze data using ontology based approach. We use various types of ontology's for opinion mining. The main task to perform by ontology is that it set the target for opinion of a product. Opinion lexicons are used which is a kind of dictionary to know about the meaning of particular word and sentence. Using the opinion lexicon, we can checked or identified the polarity of particular item. We use sentiwordNet, WordNet etc as an opinion lexicons. Then aspect based or featured based polarity is checked the review is positive, negative or natural and then sentiment aggregation or a sentiment summary means a whole product is positive, natural or negative. In this survey different approaches and methodology is used. Graph based ontology and tree based ontology are maximum use. Using the ontology fast and accurate result is found.

\section{REFERENCES}

1. Padmaja, S., \& Fatima, S. S. (2013). Opinion mining and sentiment analysis-an assessment of peoples' belief: A survey. International Journal of Ad hoc, Sensor \& Ubiquitous Computing, 4(1), 21.

2. Jagtap, V. S., \& Pawar, K. (2013). Analysis of different approaches to sentence-level sentiment classification. International Journal of Scientific Engineering and Technology, 2(3), 164-170.

3. Vivekanandan, K., \& Aravindan, J. S. (2014). Aspect-based opinion mining: A survey. International Journal of Computer Applications, 106(3).

4. Ravi, K., \& Ravi, V. (2015). A survey on opinion mining and sentiment analysis: tasks, approaches and applications. Knowledge-Based Systems, 89, 14-46.

5. Kolkur, S., Dantal, G., \& Mahe, R. (2015). Study of different levels for sentiment analysis. International Journal of Current Engineering and Technology, 5(2), 768Ü770.

6. Devika, M. D., Sunitha, C., \& Ganesh, A. (2016). Sentiment analysis: A comparative study on different approaches. Procedia Computer Science, 87, 44-49.

7. Kharde, V., \& Sonawane, P. (2016). Sentiment analysis of twitter data: a survey of techniques. arXiv preprint arXiv:1601.06971

8. Mäntylä, M. V., Graziotin, D., \& Kuutila, M. (2018). The evolution of sentiment analysis - A review of research topics, venues, and top cited papers. Computer Science Review, 27, 16-32.

9. Borth, D., Ji, R., Chen, T., Breuel, T., \& Chang, S. F. (2013, October). Large-scale visual sentiment ontology and detectors using adjective noun pairs. In Proceedings of the 21st ACM international conference on Multimedia (pp. 223-232). ACM.

10. Kreutzer, J., \& Witte, N. (2013). Opinion Mining Using SentiWordNet. Uppsala University.

11. Mukherjee, S., \& Joshi, S. (2013). Sentiment aggregation using ConceptNet ontology. In Proceedings of the Sixth International Joint Conference on Natural Language Processing (pp. 570-578).

12. Nithish, R., Sabarish, S., Kishen, M. N., Abirami, A. M., \& Askarunisa A. (2013, December). An Ontology based Sentiment Analysis for mobile products using tweets. In Advanced Computing (ICoAC), 2013 Fifth International Conference on (pp. 342-347). IEEE.

13. Singh, V. K., Piryani, R., Uddin, A., \& Waila, P. (2013, March). Sentiment analysis of movie reviews: A new feature-based heuristic for aspect-level sentiment classification. In Automation, computing, communication, control and compressed sensing (iMac4s), 2013 international multi-conference on (pp. 712-717). IEEE.

14. Cambria, E. (2013, November). An introduction to concept-level sentiment analysis. In Mexican international conference on artificial intelligence (pp. 478-483). Springer, Berlin, Heidelberg.
15. Kontopoulos, E., Berberidis, C., Dergiades, T., \& Bassiliades, N (2013). Ontology-based sentiment analysis of twitter posts. Expert systems with applications, 40(10), 4065-4074.

16. Cambria, E., Schuller, B., Xia, Y., \& Havasi, C. (2013). New avenues in opinion mining and sentiment analysis. IEEE Intelligent Systems, 28(2), 15-21.

17. Freitas, L. A., \& Vieira, R. (2013, May). Ontology based feature level opinion mining for portuguese reviews. In Proceedings of the 22nd International Conference on World Wide Web (pp. 367-370). ACM.

18. Najmi, E., Hashmi, K., Malik, Z., Rezgui, A., \& Khanz, H. U. (2014, November). ConceptOnto: An upper ontology based on Conceptnet. In Computer Systems and Applications (AICCSA), 2014 IEEE/ACS 11th International Conference on(pp. 366-372). IEEE.

19. Haider, S. Z. (2012). AN ONTOLOGY BASED SENTIMENT ANALYSIS: A Case Study

20. Lau, R. Y., Li, C., \& Liao, S. S. (2014). Social analytics: Learning fuzzy product ontologies for aspect-oriented sentiment analysis. Decision Support Systems, 65, 80-94. 